\title{
Inadvertent brain penetration during neonatal nasotracheal intubation
}

\section{Newborn Services, Department of Paediatrics, University of British Columbia, Vancouver, Canada D Cameron B A Lupton \\ Correspondence to: Dr Duncan Cameron Department of Child Health University Hospital of Wales, Heath Park, Cardiff CF4 4XW.}

Accepted 6 January 1993

\author{
D Cameron, B A Lupton
}

\begin{abstract}
During routine nasal intubation of a premature infant, the endotracheal tube penetrated the brain. Bloodstained cerebrospinal fluid and neural tissue was apparent. Initial cranial sonography was normal, but the infant later developed extensive intracranial haemorrhage. Rotation of an endotracheal tube to facilitate insertion angles the bevel at the tip upwards, increasing risk of brain penetration. Great care is required during nasal intubation; use of a small feeding tube over which to slide an endotracheal tube may be helpful.

(Arch Dis Child 1993; 69: 79-80)
\end{abstract}

The relative merits of nasal versus oral endotracheal intubation in preterm infants have long been debated. Proponents of the oral route argue that oral intubation of such infants is easier to perform, is less traumatic to the infant, is a secure method in experienced hands, and avoids the common problem of unsightly deformities of the nostril resulting from long term nasal intubation. They also argue that a larger bore endotracheal tube may be used, thus allowing more satisfactory ventilation; those who favour the nasal route point to the considerable problems in palatal deformities associated with long term oral intubation and argue that a nasal tube is much more stable with a lower rate of accidental extubation than oral tubes. ${ }^{1-3} \mathrm{~A}$ range of complications arising acutely during intubation by either route has been described, including oesophageal intubation, right mainstem bronchus intubation, local trauma to the alveolar margin with incautious use of the laryngoscope, and less commonly perforation of the larynx and bronchus. We report a case of inadvertent brain penetration during routine nasal intubation of a preterm infant. Such a complication has previously only been described in association with facial and basal skull fractures.

\section{Case report}

A boy weighing $1370 \mathrm{~g}$ was born at 29 weeks' gestation to a 29 year old healthy mother who had had two previous preterm deliveries at 31 and 34 weeks resulting in healthy children. This pregnancy had been complicated by bleeding in the first trimester and by preterm labour at 29 weeks. An emergency caesarean section was performed for transverse lie of the infant. Apgar scores were 6 at 1 minute and 8 at 5 minutes; the infant was intubated orally without difficulty with a size 3.0 endotracheal tube at 3 minutes of age for poor respiratory effort. The infant was transferred to the special care nursery and was ventilated at pressures of $21 / 4 \mathrm{~cm} \mathrm{H}_{2} \mathrm{O}$, rate $30 /$ minute, and in $100 \%$ oxygen. Clinical examination of the infant was normal apart from having a single umbilical artery; the placenta showed abnormal vessel insertion and had a central infarcted area. Transport to the regional neonatal intensive care unit was requested for subsequent care, and the infant remained stable until the arrival of the transport team at approximately 3 hours of age.

For the purposes of transport and for subsequent care of the infant it was decided to replace the oral endotracheal tube with a nasal one as is the practice of the regional unit. Accordingly, a lubricated $3.0 \mathrm{~cm}$ Portex Magill tube was introduced into the right nostril. Moderate resistance was encountered, and after clockwise rotation of the endotracheal tube to 90 degrees, the tube passed easily to a depth of $8 \mathrm{~cm}$. A laryngoscope was inserted, but the endotracheal tube was not visible in the pharynx. At this point, clear fluid and white matter was observed welling up from the endotracheal tube. It was immediately withdrawn, and an oral endotracheal tube was inserted without difficulty. The white material was removed from the endotracheal tube and was placed in normal saline for later inspection. The infant was transferred without further problems, and a cranial ultrasound scan was performed shortly after arrival at the regional centre at approximately 6 hours of age. This showed no specific abnormalities, and the cribriform plate and sphenoid ridges appeared normal. A bloodstained discharge was observed from the right nostril over the next day; considerable facial bruising evolved in this area, and prophylactic ampicillin, gentamicin, and cefotaxime were given. During the first two days of life, the infant had problems with blood pressure, with a mean of between 23-30 $\mathrm{mm} \mathrm{Hg}$ despite colloid and inotropic support. Examination of the white tissue from the nasal endotracheal tube revealed two cores of tissue, measuring 1.0 and $1.3 \mathrm{~cm}$ long with a diameter of $0.3 \mathrm{~cm}$. It was identified as neural tissue, with the larger fragment appearing consistent with cerebral cortex.

Cranial ultrasound scan on the third day of life showed bilateral subependymal haemorrhage with a mildly dilated right lateral ventricle and increased right periventricular echogenicity. A cranial computed tomogram on day five showed both lateral ventricles filled with blood and bilateral periventricular haemorrhage. The cribriform plate appeared normal 
and no nasal encephalocele was present. The infant went on to develop bronchopulmonary dysplasia, requiring ventilation until day 36 , and serial cranial ultrasound scans demonstrated the evolution of a large left porencephalic cyst and posthaemorrhagic ventricular dilatation necessitating the placement of a reservoir to allow regular cerebrospinal fluid removal. The infant's course was further complicated by ventriculitis due to Staphylococcus epidermidis that prompted the removal of the reservoir; at 6 months a ventriculoperitoneal shunt was inserted. At 8 months of age the infant was showing developmental delay with persistent head lag and generalised hypotonia.

\section{Discussion}

It seems likely that the nasal endotracheal tube passed through the back of the nose into the brain. A possible explanation for this alarming occurrence is that partial clockwise rotation of a rightsided endotracheal tube results in the bevel at its tip being angled in such a way as to allow upward progress of the endotracheal tube towards the cranial cavity. It is not unusual to encounter a degree of resistance while passing a nasotracheal endotracheal tube, and partial rotation of the tube is a manoeuvre frequently employed to guide the endotracheal tube to a correct position. The facial bones of premature infants are both thin and incompletely fused. The cribriform plate and the posterior nasal bones are difficult to image with great resolution in premature infants, and although these appeared normal, and no track of the endotracheal tube was identified, it seems more likely that the brain was penetrated rather than that a small nasal encephalocele was encountered. Traumatic penetration of the brain during nasal intubation has not previously been reported in an otherwise normal preterm infant, although it is conceivable that lesser degrees of damage to the cribriform plate and posterior nasal bones may go unnoticed during a difficult nasal intubation.

Extreme care should be exercised in the insertion of nasal endotracheal tubes in premature infants; tubes should not be rotated, and undue pressure should never be applied. When resistance is encountered the nasal endotracheal tube should be removed. In this situation, it may be helpful to insert a small feeding tube (for example 8 French gauge) to act as a guide over which gently to slide the endotracheal tube. An awareness of the fragility of the posterior nasal structures is required of all who perform this delicate procedure.

1 Black AE, Hatch DE, Nauth-Misir N. Complications of nasotracheal intubation in neonates, infants and children; a review of 4 years experience in a children's hospital. Br $\mathcal{F}$ Anaesth 1990; 65: 461-7.

2 Rivera R, Tibballs J. Complications of endotracheal intubation and mechanical ventilation in infants and children. tion and mechanical ventilation

3 Angelos GM, Smith DR, Jorgenson R, Sweeney F. Oral complications associated with neonatal oral tracheal intubation; a critical review. Paediatr Dent 1989; 11: 133-40. 